\title{
Concentrações séricas hormonais em vacas azebuadas submetidas à baixa e alta ingestão alimentar
}

\author{
Aline Carvalho Martins ${ }^{(1)}$, Marcos Rollemberg Mollo(2), Michele Ricieri Bastos ${ }^{(1)}$, Monique Mendes Guardieiro(1) \\ e Roberto Sartori ${ }^{(3)}$
}

\begin{abstract}
(1)Universidade Estadual Paulista, Faculdade de Medicina Veterinária e Zootecnia, Distrito de Rubião Júnior, s/no, CEP 18618-000 Botucatu, SP. E-mail: alinecarvalhomartins@hotmail.com, michelerbastos@gmail.com, moniqueguardieiro@yahoo.com.br (2)Universidade de Brasília, Faculdade de Agronomia e Medicina Veterinária, Caixa Postal 4508, CEP 70910-900 Brasília, DF. E-mail: marcosmollo@hotmail.com (3)Embrapa Recursos Genéticos e Biotecnologia, Caixa Postal 02372, CEP 70770-901 Brasília, DF. E-mail: sartori@cenargen.embrapa.br
\end{abstract}

Resumo - O objetivo deste trabalho foi avaliar a influência da ingestão alimentar nas concentrações séricas de hormônios reprodutivos e metabólicos em vacas azebuadas. Dezoito vacas foram divididas em dois grupos: 170\% (alta ingestão = A) e 66\% (baixa ingestão = B) da dieta de manutenção. Com 21 dias nas dietas experimentais, as vacas tiveram o estro sincronizado. Posteriormente, os ovários foram avaliados por ultra-sonografia transretal e sangue foi coletado diariamente até o dia 7 do ciclo (ovulação = dia 1). Na análise estatística, utilizou-se o teste t. As vacas ganharam $1,1 \mathrm{~kg}$ por dia no grupo A e perderam 1,5 kg por dia de PV no grupo B. Apesar de não ter havido diferença entre os grupos no diâmetro máximo do folículo ovulatório, o grupo A apresentou pico préovulatório de estradiol sérico menor. Não foi observada diferença entre os grupos quanto ao volume luteal e concentração sérica de progesterona no dia 7 do ciclo e de FSH, IGF-I e insulina séricos no período periovulatório. As dietas experimentais não alteraram a função ovariana e as concentrações séricas de hormônios reprodutivos e metabólicos, com exceção do estradiol, sugerindo que, no grupo de alta ingestão, ocorreu maior metabolismo desse hormônio.

Termos para indexação: Bos indicus, corpo lúteo, esteróides, folículo, metabolismo, nutrição.

\section{Serum hormone concentrations of zebu cows under low and high feed intake}

\begin{abstract}
The objective of this work was to evaluate the influence of feed intake on serum concentrations of reproductive and metabolic hormones in zebu cows. Eighteen cows were divided into two groups: 170\% (high feed intake $=\mathrm{H}$ ) and 66\% (low feed intake $=\mathrm{L}$ ) of the maintenance diet. Within 21 days on the experimental diets, cows had estrus synchronized. Subsequently, ovaries were scanned by trans-rectal ultrasonography and blood samples were collected daily until day 7 of the cycle (ovulation = day 1). For statistical analysis, $t$ test was utilized. Cows from group $\mathrm{H}$ gained $1.1 \mathrm{~kg}$ per day and from group Llost $1.5 \mathrm{~kg}$ per day of body weight. Although there was no difference between groups regarding maximum diameter of the ovulatory follicle, a lower preovulatory estradiol surge was observed in group H cows. There was no difference between groups for luteal tissue volume and serum progesterone concentration on day 7 and serum FSH, IGF-I and insulin concentrations during the periovulatory period. Experimental diets did not alter ovarian function and serum concentrations of reproductive and metabolic hormones, except for estradiol, suggesting that greater metabolism of this hormone has occurred on the cows under high feed intake.
\end{abstract}

Index terms: Bos indicus, corpus luteum, steroids, follicle, metabolism, nutrition.

\section{Introdução}

A nutrição afeta o processo reprodutivo de diferentes espécies e vários relatos mostram sua influência na puberdade, produção de gametas, desenvolvimento embrionário e placentário e também na lactação (Martin et al., 2004). Na espécie bovina, o sucesso da eficiência reprodutiva é dependente de um bom manejo nutricional.
A nutrição influencia a reprodução de bovinos de acordo com a condição metabólica dos animais (Adamiak et al., 2005), isto é, com a condição de balanço energético associada à quantidade de dieta ingerida.

Alterações na dieta são capazes de modificar o metabolismo dos ruminantes, alterando alguns hormônios metabólicos, entre eles GH, insulina, IGF-I e leptina, que têm grande participação na função ovariana (Webb et al., 2004). 
Além disso, animais com consumo alimentar maior apresentam maior metabolismo hepático. Isto leva à queda nas concentrações circulantes de hormônios esteróides (estradiol e progesterona) devido ao aumento na taxa de degradação hepática desses hormônios (Parr et al., 1993a, 1993b; Sangsritavong et al., 2002; Vasconcelos et al., 2003).

Estudos mostraram influência dessas alterações induzidas pela nutrição no número de folículos recrutados (Gutierrez et al., 1997; Gong et al., 2002), na taxa de crescimento folicular (Murphy et al., 1991; Bossis et al., 1999; Armstrong et al., 2001), no tamanho do folículo ovulatório (Murphy et al., 1991; Bossis et al., 1999) e do corpo lúteo (Murphy et al., 1991) e no número de ondas foliculares durante o ciclo estral em vacas (Murphy et al., 1991). Entretanto, a maioria desses estudos foi realizada com animais de raças européias.

O objetivo deste trabalho foi avaliar a influência da alta e baixa ingestão alimentar na função ovariana e nas concentrações dos hormônios reprodutivos e metabólicos em vacas aneloradas.

\section{Material e Métodos}

$\mathrm{O}$ experimento foi realizado durante os meses de outubro a dezembro de 2005 no Campo Experimental Sucupira da Embrapa Recursos Genéticos e Biotecnologia em Riacho Fundo, DF. Foram utilizadas 18 vacas aneloradas não-lactantes com escore de condição corporal (ECC) inicial de 2,8 $\pm 0,1$ (escala de 1-5; Houghton et al., 1990) e peso vivo (PV) inicial de $408,4 \pm 13,5 \mathrm{~kg}$. Todos os animais passaram por um período de adaptação à dieta de manutenção de 14 dias. Após esse período, os animais foram divididos aleatoriamente em dois grupos, em um experimento inteiramente casualizado, recebendo dietas de $170 \%$ (alta ingestão) ou 66\% (baixa ingestão) em relação à manutenção, por 35 dias.

A dieta foi elaborada e balanceada de acordo com as exigências do National Research Council (1996) para essa categoria animal e consistiu de $36,6 \%$ de feno de coast-cross, $18,3 \%$ de silagem de milho, $24,4 \%$ de milho-grão, $12,2 \%$ de farelo de trigo e $8,5 \%$ de concentrado comercial (Boião PPU, Integral Nutrição Animal, Goiânia, GO). Os animais ingeriram 1,52, 2,74 e 1,06\% em relação ao PV das dietas de manutenção, alta e baixa ingestão alimentar, respectivamente, sem haver sobras nos cochos. O concentrado forneceu $12 \mathrm{~g} \mathrm{~kg}^{-1}$ de Ca, $9 \mathrm{~g} \mathrm{~kg}^{-1}$ de $\mathrm{P}$, $1.000 \mathrm{UI} \mathrm{kg}^{-1}$ de vitamina A.
O PV e o ECC foram avaliados semanalmente e a dieta foi ajustada de acordo com o peso dos animais. As vacas foram alojadas em dois piquetes sem gramínea, onde tinham acesso à dieta oferecida, além de água e sal mineral ad libitum.

As percentagens de matéria seca (MS), nutrientes digestíveis totais (NDT) e proteína bruta (PB) dos componentes da dieta oferecida e a ingestão diária das dietas estão apresentados nas Tabelas 1 e 2, respectivamente.

Após 21 dias recebendo dietas experimentais, as vacas tiveram o estro sincronizado. Foram aplicados $50 \mu$ i.m. de GnRH (Gestran Plus, Tecnopec, São Paulo, Brasil) e colocado um implante intravaginal de progesterona (DIB, Syntex, Buenos Aires, Argentina) no dia 0. Após sete dias, retirou-se o implante e aplicaram-se $150 \mu \mathrm{g}$ i.m. de prostaglandina F2 $\alpha$ (Veteglan, Calier, São Paulo, Brasil). Desse dia até a ovulação, os ovários foram avaliados diariamente com ultra-som (Aloka SSD-500, Japão), utilizando-se uma probe linear de 7,5 MHz (Aloka, Japão) para a identificação do diâmetro máximo do folículo ovulatório. Amostras de sangue foram coletadas no período periovulatório. Seis dias após a ovulação (ovulação $=$ dia 1 do ciclo estral), foi avaliado o tamanho do corpo lúteo por meio de ultra-sonografia e o volume luteal foi

Tabela 1. Composição de matéria seca (MS), nutrientes digestíveis totais (NDT) e proteína bruta (PB) dos componentes da dieta dos animais.

\begin{tabular}{lccc}
\hline Componentes & MS & NDT & PB \\
\hline Feno coast-cross & 85,0 & 50,0 & 10,0 \\
Silagem milho & 25,0 & 62,8 & 7,5 \\
Milho & 90,0 & 80,0 & 9,0 \\
Trigo & 90,0 & 70,0 & 14,0 \\
Concentrado & 90,0 & 65,0 & 22,0 \\
\hline Média & 37,9 & 62,4 & 11,5 \\
\hline
\end{tabular}

Tabela 2. Ingestão diária de nutrientes da dieta de manutenção, de alta ( $170 \%$ da dieta de manutenção) e de baixa ingestão alimentar (66\% da dieta de manutenção).

\begin{tabular}{lccc}
\hline Dieta & Consumo de MS & $\begin{array}{c}\text { Proteína total } \\
\end{array}$ & NDT \\
\hline Manutenção & 6,3 & 0,7 & 3,9 \\
Baixa ingestão (66\%) & 4,4 & 0,5 & 2,7 \\
Alta ingestão (170\%) & 11,3 & 1,3 & 7,1 \\
\hline
\end{tabular}


estimado segundo Sartori et al. (2004). Nesse dia, também se coletou sangue para quantificação da concentração sérica de progesterona.

O sangue foi coletado por punção dos vasos coccígeos com tubos de Vacutainer, refrigerado por 24 horas e centrifugado a $1.600 \mathrm{~g}$ por $15 \mathrm{~min}$. O soro foi, então, congelado a $-20^{\circ} \mathrm{C}$, para posteriormente ser analisado para dosagem de progesterona, estradiol, insulina e FSH por radioimunoensaio (RIE) e IGF-I por ensaio imunorradiométrico (IRMA). Os kits utilizados em cada ensaio e os respectivos coeficientes de variação $(\mathrm{CV})$ estão descritos na Tabela 3.

As dosagens de progesterona sérica foram realizadas no dia 7 do ciclo estral, de estradiol sérico e de IGF-I, foram realizadas nos dias da ovulação e um e dois dias antes. As dosagens de insulina e FSH foram realizadas no dia da ovulação e um dia antes. As dosagens de IGF-I total foram realizadas após extração de acordo com as instruções no kit. Na análise de estradiol, as amostras de soro sofreram extração dupla com éter dietílico e o ensaio foi realizado utilizando-se kit com modificações de acordo com Kulick et al. (1999). A quantificação do FSH sérico foi realizada com a utilização de USDA-bFSH para iodinação e padrões de referência, e anti-soro NIDDK-anti-oFSH (Bolt \& Rollings, 1983). As variáveis estudadas nos dois grupos experimentais foram comparadas pelo teste $t$ de Student.

\section{Resultados e Discussão}

As vacas ganharam $1,1 \mathrm{~kg}$ por dia de PV no grupo de alta ingestão e perderam $1,5 \mathrm{~kg}$ por dia de $\mathrm{PV}$ no grupo de baixa ingestão, durante os 35 dias, recebendo as dietas experimentais. Apesar da significante perda de peso do grupo sob baixa ingestão alimentar, não houve

Tabela 3. Descrição dos kits utilizados e coeficientes de variação $(\mathrm{CV})$ intra-ensaio obtidos nas dosagens hormonais realizadas.

\begin{tabular}{llc}
\hline Ensaio & \multicolumn{1}{c}{ Kit } & CV (\%) \\
\hline Progesterona & Coat-a-count progesterone, & 7,5 \\
& DPC-Medlab (TKPG2) & \\
Estradiol 17ß & DSL-39100, estradiol 3 ${ }^{\underline{a}}$ geração RIA & 7,4 \\
FSH & Vide texto & 5,6 \\
Insulina & Coat-a-count insulin, & 4,8 \\
& DPC-Medlab (TKIN5) & \\
IGF-I total & DSL-5600 IRMA Active & 5,7 \\
\hline
\end{tabular}

uma queda detectável no ECC nestes animais que permaneceram com ECC entre 2,7 e 2,9 durante todo o experimento. Por sua vez, em animais do grupo de alta ingestão, o ECC elevou-se de 2,7 a 3,7 entre o início e final do período estudado.

Esperava-se, baseado em informações da literatura, que tais mudanças agudas na dieta dos animais exercessem influência na função ovariana, uma vez que sistemas hormonais que respondem a mudanças na nutrição também afetam a reprodução. Entretanto, ao se avaliar o perfil de alguns hormônios metabólicos nos animais, após três semanas nas dietas experimentais, não se detectou diferença entre os grupos nas concentrações séricas de IGF-I total e insulina durante o período peri-ovulatório (Tabela 4). Em contraste, em novilhas cruzadas (Hereford x Holandês), foi observado aumento de insulina após uma semana de ingestão de $200 \%$ da dieta de manutenção (Gong et al., 2002). Nessa mesma categoria animal, foi demonstrado aumento de insulina e IGF-I circulantes após 10 dias de fornecimento de uma dieta altamente energética (Armstrong et al., 2001). Gutierrez et al. (1997) descreveram aumento de insulina, mas não de IGF-I sérico em novilhas que receberam 200\% das exigências nutricionais para manutenção após duas semanas na dieta. Scaramuzzi et al. (2006) relataram que a infusão de glicose em ovelhas provocou aumento imediato nas concentrações sanguíneas de insulina, porém sem efeito nas concentrações plasmáticas de IGF-I. Esses autores sugeriram que o IGF-I não responde a mudanças na dieta a curto prazo. Portanto, efeitos imediatos da nutrição por meio do sistema IGF no folículo, caso ocorram, devem ser mediados por mecanismos que, provavelmente, envolvam o sistema insulina-glicose.

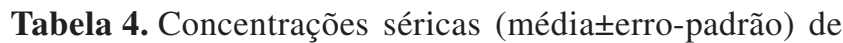
hormônios em vacas submetidas à baixa $(n=9)$ ou alta $(n=9)$ ingestão alimentar.

\begin{tabular}{|c|c|c|c|}
\hline Homônios & $\begin{array}{c}\text { Baixa } \\
\text { ingestão }\end{array}$ & $\begin{array}{c}\text { Alta } \\
\text { ingestão }\end{array}$ & $\mathrm{P}$ \\
\hline $\begin{array}{l}\text { Pico pré-ovulatório } \\
\text { de estradiol } 17 \beta\left(\mathrm{pg} \mathrm{mL}^{-1}\right)\end{array}$ & $17,2 \pm 2,5$ & $9,8 \pm 1,2$ & 0,03 \\
\hline $\begin{array}{l}\text { Progesterona no dia } 7 \\
\text { do ciclo estral (ng mL }\end{array}$ & $3,9 \pm 0,5$ & $2,6 \pm 0,6$ & 0,12 \\
\hline $\begin{array}{l}\text { FSH no período } \\
\text { peri-ovulatório }\left(\mathrm{ng} \mathrm{mL}^{-1}\right)\end{array}$ & $0,70 \pm 0,08$ & $0,62 \pm 0,04$ & 0,38 \\
\hline $\begin{array}{l}\text { IGF-I total no período } \\
\text { peri-ovulatório }\left(\mathrm{ng} \mathrm{mL}^{-1}\right)\end{array}$ & $334,9 \pm 44,0$ & $407,0 \pm 37,2$ & 0,51 \\
\hline $\begin{array}{l}\text { Insulina no período } \\
\text { peri-ovulatório }\left(\mu \mathrm{UI} \mathrm{mL}^{-1}\right)\end{array}$ & $4,7 \pm 1,5$ & $6,4 \pm 1,3$ & 0,40 \\
\hline
\end{tabular}


Alguns estudos demonstraram maior taxa de crescimento folicular ou maior diâmetro do folículo ovulatório em animais alimentados com dietas com altos níveis energéticos (Murphy et al., 1991; Armstrong et al., 2001; Sartori et al., 2002). No entanto, animais que receberam as dietas de alta e baixa ingestão alimentar neste trabalho, não diferiram no diâmetro máximo do folículo ovulatório (Tabela 5). Isso pode ser justificado por concentrações séricas de insulina e IGF-I similares entre os dois tratamentos, considerados importantes mediadores da estimulação folicular pela nutrição. Também não houve diferença entre os grupos nas concentrações séricas de FSH (Tabela 4). Essa observação corrobora outros relatos, que têm demonstrado que alterações na dieta por curto ou médio período não afetam as concentrações circulantes de FSH (O'Callaghan \& Boland, 1999; Diskin et al., 2003). A restrição energética, entretanto, parece diminuir a secreção de LH pela modulação na liberação de GnRH (McShane et al., 1993; Bossis et al., 1999; Diskin et al., 2003).

Outro mecanismo fisiológico importante nesse processo é a mudança nas concentrações de estradiol circulante, as quais podem estar reduzidas em animais com alta ingestão alimentar, como conseqüência de aumento na taxa de degradação hepática desse hormônio devido ao aumento no fluxo sanguíneo (Parr et al., 1993a, 1993b; Sangsritavong et al., 2002; Vasconcelos et al., 2003). De fato, animais com alta ingestão apresentaram menores concentrações séricas de estradiol (Tabela 4), confirmando resultados anteriores (Lopez et al., 2004; Sartori et al., 2004; Wolfenson et al., 2004). Apesar de ter ocorrido maior taxa de degradação ou menor produção de estradiol no grupo de alta ingestão, o mesmo não foi observado com a progesterona seis dias após a ovulação (Tabela 4). Pode ter havido um número

Tabela 5. Diâmetro máximo do folículo ovulatório e volume do corpo lúteo (média土erro-padrão) no dia 7 do ciclo estral em vacas submetidas à baixa $(n=9)$ ou alta $(n=9)$ ingestão alimentar.

\begin{tabular}{lccc}
\hline Variáveis & $\begin{array}{c}\text { Baixa } \\
\text { ingestão }\end{array}$ & $\begin{array}{c}\text { Alta } \\
\text { ingestão }\end{array}$ & $\mathrm{P}$ \\
\hline $\begin{array}{l}\text { Diâmetro do folículo } \\
\text { ovulatório (mm) }\end{array}$ & $12,5 \pm 0,8$ & $12,8 \pm 0,4$ & 0,78 \\
Volume do corpo lúteo $\left(\mathrm{mm}^{3}\right)$ & $2.891,6 \pm 418,2$ & $2.469,3 \pm 578,2$ & 0,58 \\
\hline
\end{tabular}

insuficiente de unidades experimentais neste trabalho que permitisse constatar diferença entre os grupos em relação à progesterona circulante.

Da mesma forma que foi observada com o diâmetro do folículo ovulatório, os grupos não diferiram quanto ao volume luteal no dia 7 do ciclo estral (Tabela 5). Essa observação diverge de estudos em que animais com maior ingestão alimentar apresentaram um maior volume luteal (Sartori et al., 2002, 2004; Wolfenson et al., 2004). Entretanto, baseando-se no fato de haver correlação positiva entre o diâmetro do folículo ovulatório e o volume luteal (Sartori et al., 2002), ao ovular folículos de tamanho semelhante, era de se esperar que as vacas, em ambos os grupos, também não divergissem em relação ao tamanho do corpo lúteo.

Em vacas de raças européias foi demonstrado que mudanças agudas na dieta foram capazes de alterar concentrações de alguns hormônios devido à mudança no estado metabólico dos animais (Adamiak et al., 2005; Freret et al., 2006). Existem fortes evidências de que a associação entre a nutrição e a função ovariana esteja relacionada principalmente com o sistema glicoseinsulina e IGF (Scaramuzzi et al., 2006). Neste trabalho, entretanto, estas inter-relações não ficaram bem definidas. Entre as possíveis razões por esta discrepância de resultados entre os estudos, deve-se destacar a raça e a subespécie dos animais utilizados neste trabalho em relação aos estudos realizados nos países de clima temperado. Desta forma, mais estudos são necessários, especialmente comparando diretamente Bos indicus a B. taurus, sobre a influência da nutrição na função reprodutiva.

\section{Conclusão}

A diferença de ingestão das dietas entre os grupos de animais não foi suficiente para alterar a função ovariana ou as concentrações séricas de hormônios reprodutivos e metabólicos, com exceção do estradiol, sugerindo que no grupo de alta ingestão, ocorreu maior metabolismo desse hormônio.

\section{Agradecimentos}

Ao Conselho Nacional de Desenvolvimento Científico e Tecnológico, pelo auxílio financeiro; à Coordenação de Aperfeiçoamento de Pessoal de Nível Superior, pela concessão de bolsa. 


\section{Referências}

ADAMIAK, S.J.; MACKIE, K.; WATT, R.G.; WEBB, R.; SINCLAIR, K.D. Impact of nutrition on oocyte quality: cumulative effects of body composition and diet leading to hyperinsulinemia in cattle. Biology of Reproduction, v.73, p.918-926, 2005.

ARMSTRONG, D.G.; MCEVOY, T.G.; BAXTER, G.; ROBINSON, J.J.; HOGG, C.O.; WOAD, K.J.; WEBB, R. Effect of dietary energy and protein on bovine follicular dynamics and embryo production in vitro: associations with the ovarian insulin-like growth factor system. Biology of Reproduction, v.64, p.1624-1632, 2001.

BOLT, D.J.; ROLLINS, R. Development and application of a radioimmunoassay for bovine follicles-stimulating hormone. Journal of Animal Science, v.23, p.263-271, 1983.

BOSSIS, I.; WETTEMANN, R.P.; WELTY, S.D.; VIZCARRA, J.A.; SPICER, L.J.; DISKIN, M.G. Nutritionally induced anovulation in beef heifers: ovarian and endocrine function preceding cessation of ovulation. Journal of Animal Science, v.77, p.1536-1546, 1999.

DISKIN, M.G.; MACKEY, D.R.; ROCHE, J.F.; SREENAN, J.M. Effects of nutrition and metabolic status on circulation hormones and ovarian follicle development in cattle. Animal Reproduction Science, v.78, p.345-370, 2003.

FRERET, S.; GRIMARD, B.; PONTER, A.A.; JOLY, C.; PONSART, C.; HUMBLOT, P. Reduction of body-weight gain enhances in vitro embryo production in overfed superovulated dairy heifers. Reproduction, v.131, p.783-794, 2006.

GONG, J.G.; ARMSTRONG, D.G.; BAXTER, G.; HOGG, C.O.; GARNSWORTHY, P.C.; WEBB, R. The effect of increased dietary intake on superovulatory response to FSH in heifers. Theriogenology, v.57, p.1591-1602, 2002.

GUTIERREZ, C.G.; OLDHAM, J.; BRAMLEY, T.A.; GONG, J.G.; CAMPBELL, B.K.; WEBB, R. The recruitment of ovarian follicles is enhanced by increased dietary intake in heifers. Journal of Animal Science, v.75, p.1876-1884, 1997.

HOUGHTON, P.L.; LEMENAGER, R.P.; HENDRIX, K.S.; MOSS, G.E.; STEWART, T.S. Effects of body composition, preand postpartum energy intake and stage of production on energy utilization by beef cows. Journal of Animal Science, v.68, p.1447-1456, 1990.

KULICK, L.J.; KOT, K.; WILTBANK, M.C.; GINTHER, O.J. Follicular and hormonal dynamics during the first follicular waves in heifers. Theriogenology, v.52, p.913-921, 1999.

LOPEZ, H.; SATTER, L.D.; WILTBANK, M.C. Relationship between level of milk production and estrous behavior of lactating dairy cows. Animal Reproduction Science, v.81, p.209-223, 2004.

MARTIN, G.B.; RODGER, J.; BLACHE, D. Nutritional and environmental effects on reproduction in small ruminants. Reproduction, Fertility and Development, v.16, p.491-501, 2004. MCSHANE, T.M.; PETERSEN, S.L.; MCCRONE, S.; KEISLER, D.H. Influence of food restriction on neuropeptide-Y, proopiomelanocortin, and luteinizing hormone - releasing hormone gene expression in sheep hypothalami. Biology of Reproduction, v.49, p.831-839, 1993.

MURPHY, M.G.; ENRIGHT, W.J.; CROWE, M.A.; MCCONNELL, K.; SPICER, L.J.; BOLAND, M.P.; ROCHE, J.F. Effect of dietary-intake on pattern of growth of dominant follicles during the oestrous-cycle in beef heifers. Journal of Reproduction and Fertility, v.92, p.333-338, 1991.

NATIONAL RESEARCH COUNCIL. Nutrient requirements of beef cattle. $7^{\text {th }}$ ed. Washington: National Academy Press, 1996. 242p.

O'CALLAGHAN, D.; BOLAND, M.P. Nutritional effects on ovulation, embryo development and the establishment of pregnancy in ruminants. Animal Science, v.68, p.299-314, 1999.

PARR, R.A.; DAVIS, I.F.; MILES, M.A.; SQUIRES, T.J. Feedintake affects metabolic-clearance rate of progesterone in sheep. Research in Veterinary Science, v.55, p.306-310, 1993a.

PARR, R.A.; DAVIS, I.F.; MILES, M.A.; SQUIRES, T.J. Liver blood flow and metabolic clearance rate of progesterone in sheep. Research in Veterinary Science, v.55, p.311-316, 1993 b.

SANGSRITAVONG, S.; COMBS, D.K.; SARTORI, R.; ARMENTANO, L.E.; WILTBANK, M.C. High feed intake increases liver blood flow and metabolism of progesterone and estradiol 17 beta in dairy cattle. Journal of Dairy Science, v.85, p.2831-2842, 2002.

SARTORI, R.; HAUGHIAN, J.M.; SHAVER, R.D.; ROSA, G.J.M.; WILTBANK, M.C. Comparison of ovarian function and circulating steroids in estrous cycles of Holstein heifers and lactating cows. Journal of Dairy Science, v.87, p.905-920, 2004.

SARTORI, R.; ROSA, G.J.M.; WILTBANK, M.C. Ovarian structures and circulating steroids in heifers and lactating cows in summer and lactating and dry cows in winter. Journal of Dairy Science, v.85, p.2813-2822, 2002.

SCARAMUZZI, R.J.; CAMPBELL, B.K.; DOWNING, J.A.; KENDALL, N.R.; KHALID, M.; MUÑOZ-GUTIÉRREZ, M.; SOMCHIT, A. A review of the effects of supplementary nutrition in the ewe on the concentrations of reproductive and metabolic hormones and the mechanisms that regulate folliculogenesis and ovulation rate. Reproduction, Nutrition and Development, v.46, p.339-354, 2006.

VASCONCELOS, J.L.M.; SANGSRITAVONG, S.; TSAI, S.J.; WILTBANK, M.C. Acute reduction in serum progesterone concentrations after feed intake in dairy cows. Theriogenology, v.60, p.795-807, 2003.

WEBB, R.; GARNSWORTHY, P.C.; GONG, J.G.; ASRMSTRONG, D.G. Control of follicular interactions and nutritional influences. Journal of Animal Science, v.82, p.E63-E74, 2004. (Suplement).

WOLFENSON, D.; INBAR, G.; ROTH, Z.; KAIM, M.; BLOCH, A.; BRAW-TAL, R. Follicular dynamics and concentrations of steroids and gonadotropins in lactating cows and nulliparous heifers. Theriogenology, v.62, p.1042-1055, 2004. 